\title{
Contamination Control Technology Study for Achieving the Science Objectives of Life-Detection Missions
}

\author{
Study Team: \\ Chris McKay, NASA ARC, (650)604-6864, Chris.McKay@NASA.gov (submitting author) \\ Alfonso Davila, NASA ARC, alfonso.davila@nasa.gov \\ Jennifer Eigenbrode, NASA GSFC, jennifer.l.eigenbrode@nasa.gov \\ Chris Lorentson, NASA GSFC, charles.c.lorentson@nasa.gov \\ Rob Gold, JHU/APL, robert.gold@jhuapl.edu \\ John Canham, Northrop Grumman/NASA GSFC, john.canham@ngc.com \\ Anthony Dazzo, KBR Inc./NASA GSFC, anthony.o.dazzo@nasa.gov \\ Therese Errigo, NASA GSFC, therese.errigo-1@nasa.gov \\ Faith Kujawa, JHU/APL, Faith.Kujawa@jhuapl.edu \\ Dave Kusnierkiewicz, JHU/APL, Dave.Kusnierkiewicz@jhuapl.edu \\ Charles Sandy, ILC Dover, crsandy @ilcdover.com \\ Erich Schulze, JHU/APL, Erich.Schulze@jhuapl \\ Antonios Seas, NASA GSFC antonios.a.seas@nasa.gov
}


Summary: This white paper summaries technological advances in science-required contamination-control engineering for in situ and sample-return life-detection missions in the Solar System. Key study results are:

1) New spacecraft barrier design that accommodates MMRTGs, is cleanable, and is repairable.

2) Purge gas cleanliness of 1 part per trillion $\mathrm{HC}$ impurity limit is feasible.

3) The barrier reduces particle contamination (likely biological) from fairing to spacecraft by $10^{-2}-10^{-3}$.

4) Spacecraft surfaces protected by barrier are 10-20x cleaner after launch than before launch.

5) In-flight bake-out of critical surfaces significantly reduced molecular contamination (by $10^{-6}$ to $10^{-12}$ ). Implementing a full spacecraft barrier, collector cover and purge, and in-flight cleaning steps will achieve cleanliness levels required of science instruments (down to femtomolar levels of biomolecules). This paper summarizes the study and lays out a set of actionable findings that support technological readiness in mitigating forward contamination risks and provide contamination transport awareness that is essential for the success of missions seeking to explore the biological potential of planetary bodies beyond Earth.

\section{Preamble - Contamination Control for Science}

Contamination control in planetary mission concepts has largely or completely focused on meeting planetary protection (PP) requirements, which involves ascertaining and verifying required levels of bioburden (viable microbes or spore counts) on spacecraft surfaces. Ultimately, life-detection missions must demonstrate reduction of viable microorganisms on the surface to a level specified by the COSPAR Planetary Protection rules, such that the requirements depend on the probability that the spacecraft will impact a region that could support microbial growth (1). Less attention is often given to possible introduction of contaminants, especially chemical species, in the sampling chain at concentrations that might interfere with life detection determinations (2). Demonstrating an effective mitigation approach for the latter can be challenging as 1) improved instrument sensitivity and resolution, possibly combined with small sample sizes or transfer efficiencies to instruments, impose stringent requirements on science contamination control, 2) there is no standard approach and it needs to be tailored to specific mission design, 3) traceability of possible contaminants to specific measurement requirements involves assumptions about planetary conditions that are often unknown and poorly understood, 4) methods for verifying the mitigation processes are often inadequate or require the spacecraft instruments (not always an option).

The next decade of planetary exploration will witness growing interest to search for evidence of extraterrestrial life. Many life detection mission concepts seek chemical signatures of life, both organic and inorganic (3-7). Stringent science-derived contamination requirements necessitate new mitigation approaches that minimize, protect from, and prevent contamination of critical surfaces of the science payload in order to enable successful life-detection missions.

\section{Study Introduction}

This technology development study was funded by NASA New Frontiers program "to develop techniques that limit spacecraft contamination and thereby enable life detection measurements on cost-capped missions" (8). Methods for cleaning and protecting the critical surfaces of the sample path responsible for collection, processing, and detections exist and are commonly in practice. However, of particular concern for upcoming life-detection missions is the high probability of particles and organic molecules transfer from the rocket fairing (provided by vendor) to the spacecraft (controlled by NASA), and for those contaminants to directly or indirectly transfer to critical surfaces influencing life-detection measurements. Launch vehicle 
processing and launch impose the greatest risk to contamination for all missions (e.g., inadvertent contaminant transfer from personnel working in the fairing, limited cleanliness monitoring and recleaning capability, contamination events, single stream environmental systems, pressurization failures, launch window limitations). Modifying launch vehicle processing to prevent such contamination is both expensive and introduces other risks. How do we achieve ultra-low contamination levels required by science given the launch processing environment?

Each life-detection mission concept will be unique based on destination, nature of the targeted samples, sampling methods, target compounds, and operations. It was beyond the scope of this technology study to address all of these possibilities; however, some of the study's innovations are widely applicable to all cases and could be leveraged in mission designs. The reference mission used for this study presses for the most stringent requirements, making it the perfect mission to develop contamination prevention and mitigation strategies. The reference mission consists of an orbiter that flies through the plume of Enceladus multiple times at low orbit to collect and analyze samples of ocean material. When necessary, specific mission design features (e.g., plume collection system, spacecraft configuration, etc.) were based on the ELSAH mission concept (9). Cassini mission results were used to constrain plume and ice particle characteristics. The primary targeted compounds for life detection are amino acids and lipids. Minimal sample volume collected is assumed to be a few microliters. Required limits of detections (LoDs) are assumed to be at the femtomolar level for each molecular structure of interest. In this case, and based on Earth-life biochemistry, these required LoDs are equivalent to single cell quantities of molecules. While a signal to noise ratio of 3:1 is desired, given the unknowns in the chemistry of collected ice particles, contamination requirements are essentially at the same level as the LoD for all potentially interfering compounds as the required instrument LoDs. How do we achieve the femtomolar contamination levels required by science for the given reference mission operations?

\section{Life-Detection Missions Require Non-Traditional Thinking}

For missions seeking to understand the biological potential of other bodies in the Solar System, science contamination requirements should be traceable to Level- 1 science requirements and mission implementation. If the contamination levels acceptable at operations are stringent, then mission design and implementation must also include verification and validation of contamination control measures taken during build, integration, launch processing, launch, and cruise will achieve the requirements for science operations.

Traditionally, we determine what cleanliness requirements we need to meet at launch to ensure the instrument performs adequately throughout the mission (Fig. 1). To achieve this, we make sure the contaminant levels that we can verify (with tape lifts, NVR swabs, visual inspections, witness samples, etc.) will not contribute enough contamination to the sample collector, processing pathways, and instrument detectors to degrade performance. Then we protect critical surfaces of the sample pathway from launch operations, and inflight outgassing/particulate transfer (covers, purges, cleaning of the fairing, environmental controls). There is no direct measure of contaminant transfer. It is assumed that particulate and molecular contamination types each have a single specific impact on science. There is no evaluation of different types of particles or molecules. Despite the effectiveness of traditional, well-proven techniques and 
operations for other mission scenarios, for life-detection missions in which acceptable contamination is at extremely low levels, traditional methods are insufficient for proper validation and verification of requirements. Verification is inadequate when the instruments of the payload offer the only verification testing available. Furthermore, traditional modelling only tracks pressure/temperature-driven evaporation and deposition of bulk contamination using view factors and simple ray tracing that computationally presents an intractable problem for low levels of molecules and particulates.

Life-detection mission designs need improved analysis techniques to evaluate contamination transfer for surfaces having monolayer ore less levels of contaminants, where current methods fail. The behavior of molecules on surfaces are different from molecules in bulk. Innovations in hardware protection/cleaning must occur to address shortcomings of traditional contamination control engineering for life-detection missions, in order to meet the stringent contamination requirements imposed by science.

In contrast to the traditional engineering approach, this technology study adopts the perspective of determining what feasible contamination control mitigation steps, including the contamination requirements at launch, are needed to meet the stringent science-derived contamination requirements at and during operations. This engineering approach relies on modeling specific molecular and particulate contamination transport from/to launch hardware, spacecraft and sample collector surfaces during each mission phase. The computational code models transport of molecular and particulate contaminants with considerations for aerodynamic, electrostatic, and gravitational forces that vary spatially and temporally, complex spacecraft geometries, and detailed surface chemistry and physics to provide accurate contamination predictions for very low levels (monolayer to sub monolayer) of contamination transport, adsorption and desorption. This approach enables adaptation of a set of mitigation strategies in mission design to ensure that the cleanliness requirements post launch are both feasible and achievable. For the reference mission, traditional mitigation protocols including a cover on the sample collector and purging with $\mathrm{N}_{2}$ gas, did not meet the science contamination requirements.

The aims of this technology study were to: 1) design and test a spacecraft barrier for cost-capped lifedetection missions, 2) investigate how contaminants transfer from fairing to spacecraft to sample-handling payload, 2) quantitatively evaluate the effectiveness of mitigation approaches.

\section{Spacecraft Barrier Technology Development}

Protocols exist for cleaning hardware surfaces and protecting them from recontamination, however, the most reliable and generally cost-effective solutions to prevent recontamination is to provide a physical barrier purged with ultra-clean gas until $\mathrm{T}_{0}$. In the case of launch processing and launch there are many uncontrolled variables, hardware ownership, and qualification issues that challenge control over recontamination of an exposed spacecraft. The Viking mission reached the same conclusion. In this study, combined mechanical and contamination engineering produce a new

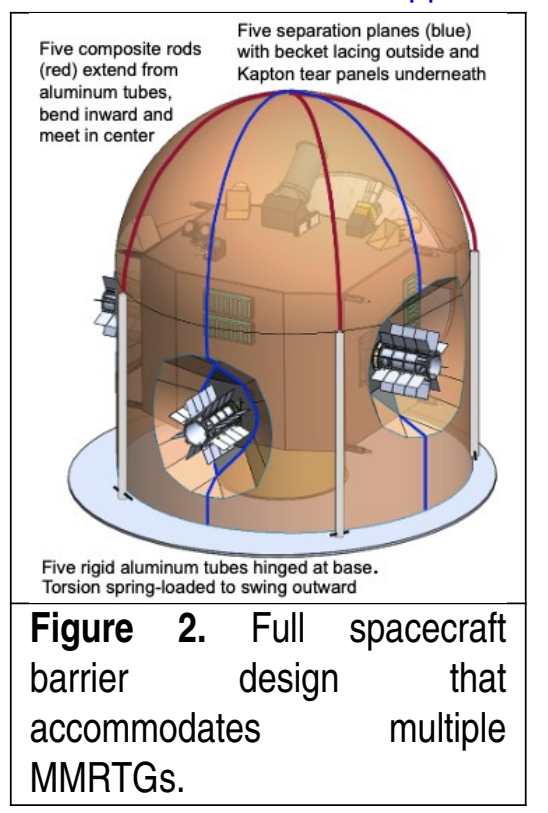


barrier technology that mechanically protects the spacecraft through launch and is readily cleanable and repairable during launch processing.

The resulting design is shown in Figure 2. The design had a number of requirements including: minimal contact with spacecraft to prevent damage; minimal leakage of purge gas; a nonoutgassing inner surface that can be precision cleaned for molecules and particulates (including bioburden); repairability without introducing additional contaminants; installation on the spacecraft in a clean room; ability to withstand pressure differentials, vibration, acoustics, and thermal environments during testing and launch; supports MMRTG's to be mounted while on the launch pad, when applicable; minimizing launch mass as much as possible; and adaptability in the design for other spacecraft. The inner surface of the barrier is smooth with minimal surface features, allowing the barrier to be readily cleaned to extreme levels. The barrier opening and release will occur after fairing separation during ascent. A reduced scale spacecraft and barrier model were constructed to test opening (Fig. 3). The model behaved as designed, providing clean separation of the barrier from the spacecraft and the MMRTG connection base.
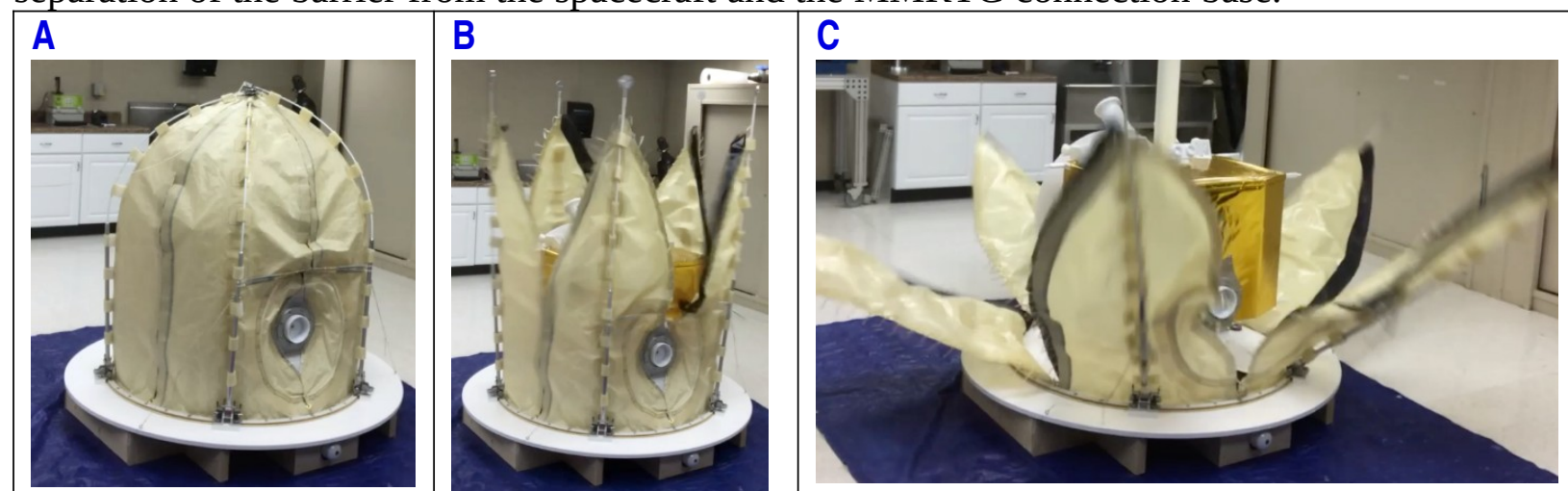

Figure 3. Time sequence of barrier opening, post fairing release. The ring on right side of barrier represents the base of an MMRTG attached to the spacecraft. A) Pre-release. B) After release, the barrier separates along engineered separation lines. This is characterized by a rate limited opening to limit particle redistribution. C) Post opening but prior to spacecraft separation from the launch adaptor and barrier.

\section{Contamination Transport Modeling Technology Development}

With respect to contamination transfer modeling, only contaminants that will make it to the instrument inlets and interfere with the measurement of sample composition, required contaminant transfer modeling. Evaluation of purge gas cleanliness concluded that ultra-clean $\mathrm{N}_{2}$ purge gas having 1 part per trillion concentrations of hydrocarbon impurities that are relevant to the reference mission is feasible. This purge of collector and barrier was used in models. Further, the models assumed fairing and spacecraft cleanliness traditionally required at launch and do not consider off-nominal or event conditions, though these could be explored in future calculations. High-fidelity computational fluid dynamics of the rocket depressurizing and venting during launch is shown in figure 4 with and without a barrier. Note the purge of the barrier (red streak on right image) helped in the removal of contaminants from the barrier/spacecraft zone and responsible for the unexpected model result of 10-20x reduction in contaminants on some parts of the spacecraft before barrier release. Figure 5 shows the resultant $10^{-2}$ to $10^{-3}$ particle reduction on spacecraft surfaces after ascent when a barrier is used. 
Science Contamination Control Technology Study

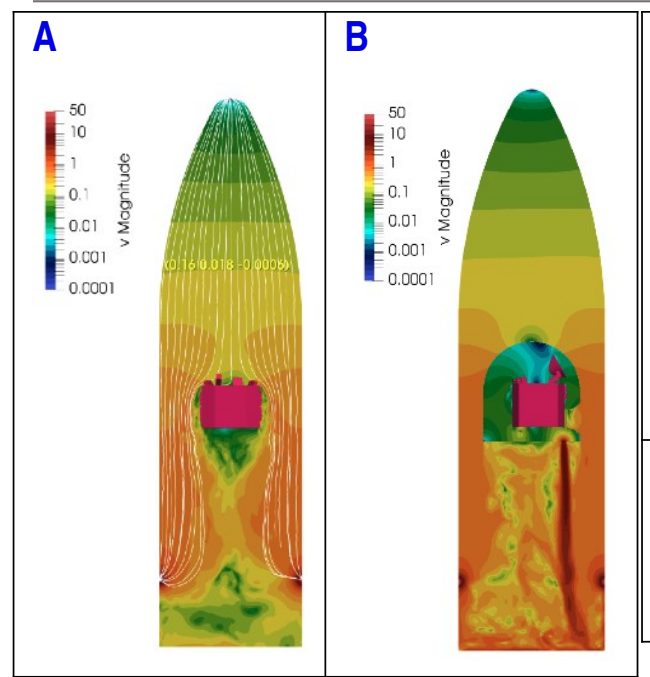

Figure 4. Computational fluid dynamics models showing launch depressurization venting flow surrounding the spacecraft with (right) and without (left) the barrier.

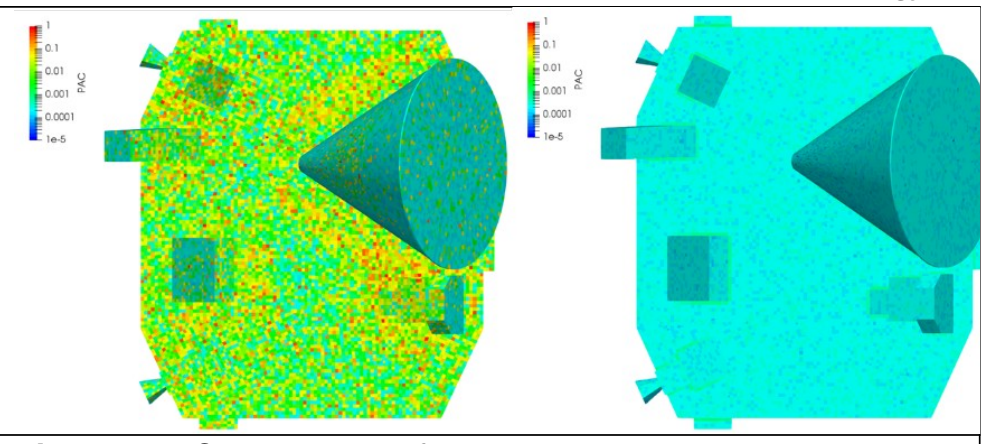

Figure 5. Comparison of the particle distributions on the spacecraft after ascent. It is worth noting that the barrier case (right) some of the surface locations are actually cleaner than before launch due to the loss of particles from the surfaces.

For the sample collector, the transfer efficiency of each contaminant on the collector's surface to the instrument inlet took into consideration an expected sample volume, ice-particle size, and ice-particle impingement energy. This allowed the requirement for the surface cleanliness at the time of sample collection to be determined.

Adsorption-desorption behavior of molecules on surfaces was subject to a literature review. Since not all molecules of interest for key science measurements were reported, representative molecules have behavior similar to target molecules were used.

The cruise mission phase of the reference mission included a $300^{\circ} \mathrm{C}$ thermal bakeout of the collector. A high-fidelity simulation of this bake-out verified the efficiency of this in-flight cleaning step for reducing molecular and particulate contaminants with an astonishing $10^{-12}$ reduction in contaminants.

\section{Actionable Findings for Meeting Stringent Contamination Requirements of Life-Detection Missions}

1. Requirement Traceability and Validity for Operations: Acceptable levels and types of contamination from the start to end of operations science operations should flow down from level-1 science and mission requirements and be validated to ensure success of the mission meeting life-detection related science objectives. This effort should start as part of mission concept development.

2. Requirement Traceability and Validity for Launch: Contamination requirements for spacecraft surfaces at launch must be traceable back to contamination requirements for operations. Recontamination occurs at different rates in different facilities and under different conditions and may vary over time. Detailed evaluation and validation of requirements, as well as mitigation approaches should be determined early in the mission design.

3. Mission Level Contamination Engineering: Due to the unprecedentedly low detection limits and the required control of false positives in life detection, it is necessary that contamination awareness and control be addressed in detail at mission concept and carried throughout the mission phases. Although time and resource intensive, verification and validation of contamination requirements is necessary prior to System Requirements Review.

4. Modeling physics: Standard model assumptions that rely on bulk-transfer physics, view geometries, and single ray tracing diverge from actual contaminant behavior in extremely 
clean systems. Higher fidelity models are necessary to understand contaminant transfer for "clean" spacecraft, such as those of life-detection missions, for launch through end of mission.

5. Modeling contaminant behavior: Modeling of contamination transport should include the chemical and physical behavior of molecules and particulates that best match contaminants that may interfere with science measurements. These could be target compounds in biosignatures, compounds known to interfere with target compound detection, and particulates that mask detection of cells or related biogeochemistry. Science-derived selection of contaminants and their attributes that are applied in models will enable mission success. Experimental validation of models, assumptions and model inputs should be carried out to assure that the modeling converges with actual behavior.

6. Experimental data needed for biological particulate contaminants: Experimental data is needed to characterize the distribution of microbial and biological contamination in terms of total viable and non-viable biological burden in cleanrooms and on space flight hardware is needed. There is very limited data concerning non-culturable bioburden. Routine quantitative evaluation of viable and non-viable biological contamination levels and rates of contamination in integration and testing facilities, and on actual hardware is needed to evaluate the risk of these contaminants to life detection missions.

7. Launch processing facilities: Given the small fraction of highly contamination sensitive launches, launch processing facilities are primarily designed to have minimal impact on other programs. More sensitive missions must address their own more stringent requirements by supplying additional funding to the launch provider, and/or utilizing additional mitigation strategies.

8. Hardware design: All spacecraft hardware design and operations (not just the science payload and operations) should respond to thoughtful consideration that enable extreme cleanliness levels to be attained. Hardware should be designed to tolerate extremely stringent cleaning processes. Geometries, surface finishes, modularity, assembly order and materials choices, can either enable or prevent achievement of required cleanliness levels.

9. Spacecraft design: Sample collection devices should not have a "view" of other spacecraft surfaces. Modeling indicates that the position of collectors is critical to mitigating molecular and particulate contamination transfer from spacecraft to collectors.

10. Flight covers: Implementing flight covers of critical surfaces, such as sample collectors, is absolutely essential for mitigating contamination transfer to critical surfaces of the sample path. The stringent contamination requirements for operations of the design reference mission were only met by including a flight cover over the sample collector. Verification of the efficacy of flight covers using high-fidelity models for the spacecraft conditions is critical.

11. Spacecraft barrier: Implementing a spacecraft barrier designed to mitigate contamination transfer from launch vehicle interior environment to spacecraft greatly reduces the level of work on the launch vehicle and the risk of contaminating the hardware.

12. Inflight cleaning: Inflight maintenance of cleanliness is a high value effort that should be incorporated into life-detection mission. Inflight, high temperature $\left(300^{\circ} \mathrm{C}\right)$ bakeout of critical surfaces is very effective at lowering molecular and biological particulate contamination levels. (At the inflight bakeout temperature, biological particles will be significantly degraded, effectively decreasing the potential for false positives.) For the design 
reference mission, the inflight bake out of the collector is essential for meeting stringent contamination requirements for science operations of the life-detection mission.

\section{Conclusions}

Life detection missions, particularly those that focus on chemical evidence of life (organic and inorganic), require stringent contamination control protocols. The stringent contamination control requirements needed for life detection missions (including cost-capped ones) can only be achieved through clearly defined science requirements, contamination transport models, model verification, hardware and spacecraft design that consider contamination control from inception, use of flight covers and spacecraft barriers, and inflight cleaning. Use of barriers, flight covers, and inflight cleaning operations greatly decrease the level of effort, cost, and risk of recontamination of the critical surfaces. Protocols to minimize contamination must be implemented at every stage of a life-detection mission, but particularly for launch processing. Life detection mission hardware must be protected following Planetary Protection compliance verification, through launch processing, through separation from the launch vehicle. Protocols to control contamination during this period need to be developed during mission design.

This technology study made key advances in barrier design as well as contaminant transport modeling that provide a means for understanding how effective mitigation strategies are and assessing what combination is best suited to meeting the science requirements of the mission. The results presented are specific to the reference mission but have applicability to any life detection missions. What is more, the technology developments of this study do not preclude the need for positive and negative control tests in mission operations. For validation of positive life detection measurements, it is required that actual negative controls be used to characterize the signals due to the measurement system. It is necessary to include positive controls in order to account for mechanisms that would otherwise lead to false negative conclusions. Still, this study demonstrates that implementing a full spacecraft barrier, collector cover with ultra-clean purge, and in-flight cleaning steps achieve cleanliness levels required of life-detection related science instruments (down to femtomolar levels of biomolecules).

\section{References}

1. COSPAR Policy on Planetary Protection, Space Research Today, No 208, August 2020, Pages 10-22. See Rules.

2. See above; for category IVB and V missions in which life detection is being carried out.

3. Williford, K., et al. (2018) The NASA Mars 2020 Rover Mission and the Search for Extraterrestrial Life. In From Habitability to Life on Mars, Elsevier, pp. 275-308. doi: 10.1016/B978-0-12-809935-3.00010-4

4. Turtle, E. P., et al. (2018) 'Dragonfly: In Situ Exploration of Titan’s Organic Chemistry and Habitability', 49th Lunar and Planetary Science Conference. doi: 10.1109/AERO.2013.6497165.

5. Eigenbrode, J., et al. (2018). Searching for Life in an Ocean World: The Enceladus Life Signatures and Habitability (ELSAH) mission concept. 42nd COSPAR Scientific Assembly. Held 14-22 July 2018, in Pasadena, California, USA, Abstract id. F3.6-3-18.

6. Reh, K. et al. (2016) 'Enceladus Life Finder: The search for life in a habitable Moon', in IEEE Aerospace Conference Proceedings. doi: 10.1109/AERO.2016.7500813. 
7. McKay, C. P. et al. (2013) 'The Icebreaker Life Mission to Mars : A Search for Biomolecular Evidence for Life', Astrobiology, 13(4), pp. 334-354. doi: 10.1089/ast.2012.0878.

8. NASA Invests in Concept Development for Missions to Comet, Saturn Moon Titan. NASA News. 20 December 2017. https://www.nasa.gov/press-release/nasa-invests-in-conceptdevelopment-for-missions-to-comet-saturn-moon-titan

9. Eigenbrode, J., et al. (2018). Searching for Life in an Ocean World: The Enceladus Life Signatures and Habitability (ELSAH) mission concept. 42nd COSPAR Scientific Assembly. Held 14-22 July 2018, in Pasadena, California, USA, Abstract id. F3.6-3-18. 